\title{
Establishing the prevalence of common, clinically relevant tissue-specific autoantibodies following SARS CoV-2 infection
}

\author{
A. G. Richter ${ }^{1}$, Adrian Shields ${ }^{1}$, Abid Karim ${ }^{1}$, David Birch ${ }^{1}$, Sian Faustini ${ }^{1}$, Lora \\ Steadman ${ }^{1}$, Kerensa Ward ${ }^{1}$, Tim Plant ${ }^{1}$, Gary Reynolds ${ }^{2}$, Tonny Veenith ${ }^{3}$, A \\ Cunningham ${ }^{4}$, M Drayson ${ }^{5}$, and David Wraith ${ }^{1}$ \\ ${ }^{1}$ University of Birmingham \\ ${ }^{2}$ NIHR \\ ${ }^{3}$ University Hospitals Birmingham NHS Foundation Trust \\ ${ }^{4}$ Birmingham University Medical School \\ ${ }^{5}$ University of Birmingham Medical School
}

April 28, 2021

\begin{abstract}
COVID-19 has been associated with both transient and persistent systemic symptoms that do not appear to be a direct consequence of viral infection. The generation of autoantibodies has been proposed as a mechanism to explain these symptoms. To understand this phenomenon in more detail, we investigated the frequency and specificity of clinically relevant autoantibodies in 84 individuals previously infected with SARS-CoV-2, suffering from COVID-19 of varying severity in both the acute and convalescent setting. These were compared with results from 32 individuals who were on ITU for non COVID reasons. We demonstrate a higher frequency of autoantibodies in the COVID-19 ITU group compared with non-COVID-19 ITU disease control patients and that autoantibodies were also found in the serum 3-5 months post COVID-19 infection. Non-COVID patients displayed a diverse pattern of autoantibodies; in contrast, the COVID-19 groups had a more restricted panel of autoantibodies including skin, skeletal muscle and cardiac antibodies. Our results demonstrate that severe COVID-19 induces a pattern of autoantibodies that may correlate with and contribute to the immune pathology associated with the long-term sequelae of infection.
\end{abstract}

Establishing the prevalence of common, clinically relevant tissue-specific autoantibodies following SARS CoV2 infection

Alex G. Richter ${ }^{1}$, Adrian M. Shields ${ }^{1}$, Abid Karim ${ }^{1}$, David Birch ${ }^{1}$, Sian E. Faustini ${ }^{1}$, Lora Steadman ${ }^{2}$, Kerensa Ward ${ }^{1}$, Timothy Plant ${ }^{1}$, Gary Reynolds ${ }^{2}$, Tonny Veenith ${ }^{3}$, Adam F. Cunningham ${ }^{2}$, Mark T. Drayson $^{1}$, David C. Wraith ${ }^{2}$

Clinical Immunology Service, Institute for Immunology and Immunotherapy, University of Birmingham, Birmingham, UK

Institute of Immunology and Immunotherapy, University of Birmingham, UK

Department of Critical Care Medicine, University Hospitals Birmingham NHS Trust, Birmingham, UK

Key words: COVID-19, SARS-CoV-2, autoimmunity, autoantibodies, Long COVID

Word count:

Number of figures: 2 
Number of tables: 2

Supplementary data: 2 tables

\title{
Correspondence to:
}

Professor Alex Richter

Clinical Immunology Service

Institute for Immunology and Immunotherapy

University of Birmingham

Birmingham, UK

a.g.richter@bham.ac.uk

\begin{abstract}
COVID-19 has been associated with both transient and persistent systemic symptoms that do not appear to be a direct consequence of viral infection. The generation of autoantibodies has been proposed as a mechanism to explain these symptoms. To understand this phenomenon in more detail, we investigated the frequency and specificity of clinically relevant autoantibodies in 84 individuals previously infected with SARS-CoV-2, suffering from COVID-19 of varying severity in both the acute and convalescent setting. These were compared with results from 32 individuals who were on ITU for non COVID reasons.

We demonstrate a higher frequency of autoantibodies in the COVID-19 ITU group compared with nonCOVID-19 ITU disease control patients and that autoantibodies were also found in the serum 3-5 months post COVID-19 infection. Non-COVID patients displayed a diverse pattern of autoantibodies; in contrast, the COVID-19 groups had a more restricted panel of autoantibodies including skin, skeletal muscle and cardiac antibodies. Our results demonstrate that severe COVID-19 induces a pattern of autoantibodies that may correlate with and contribute to the immune pathology associated with the long-term sequelae of infection.
\end{abstract}

\section{Introduction}

Infection is a common event that can disrupt immunological tolerance and, in some circumstances, lead to autoimmune disease [1].Viral infections have been linked to both the initiation of a range of autoimmune diseases and disease relapse in individuals with existing conditions [2]. For most autoimmune diseases, it is not clear whether infection is the sole precipitating event, an inevitable consequence of a genetic predisposition or whether infection is a necessary trigger in a genetically susceptible individual.

Early data suggest that autoimmune phenomena may exacerbate the immune pathology associated with SARS-CoV-2 infection or trigger long-term autoimmune complications secondary to bystander activation or molecular mimicry. There are reports of SARS-CoV-2 infection being associated with a number of autoimmune disorders including Guillain-Barre Syndrome (GBS) [3] and various cytopenias [4]. Anti-phospholipid antibodies have been detected in $~ 50 \%$ of hospitalised patients and linked to an increased incidence of cerebral infarction; however, the clinical relevance of this observation in COVID-19 remains controversial as anti-phospholipid antibody generation in acute illness is a common, non-specific finding [5-7]. Also, neutralising antibodies against type 1 anti-viral cytokines, Interferon (IFN)- $\omega$ and/or IFN- $\alpha$ have been found in over $10 \%$ of patients with COVID-19 pneumonia [8]. By screening a yeast expression library, Wang et al. identified autoantibodies against cytokines (including type 1 IFNs), CNS antigens and extracellular matrix proteins whose frequency correlated with disease severity [9].

Paediatric multisystem Inflammatory Syndrome (PIMS-TS) is a rare condition that occurs as a late complication of SARS-CoV-2 infection. Children suffering from this post-COVID inflammatory condition were also seropositive for anti-endothelial antibodies that may contribute to their pathology [10-12]. We and others 
are now searching for tissue specific autoantibodies in adults. Kreye and colleagues screened for CNS autoantibodies using murine brain sections identifying anti-Yo and NMDA-R as well as a variety of antibodies against epitopes including vessel endothelium [12]. Evidence has arisen that some of these antibodies may arise through cross-reactive recognition of self-antigens by antibodies specific for SARS-CoV-2[13].

Identifying the relationship between autoimmune antibody induction and COVID-19 is further complicated by the spectrum of presentations of this disease. In particular, the severity of disease may require hospitalisation and the more severe presentations of disease may influence the maintenance of autoantibodies during convalescence. To investigate any potential links between SARS-CoV-2 infection and autoantibodies we examined sera from acute and convalescent COVID-19 patients, some of whom had been hospitalised, for the presence of autoantibodies to a spectrum of antigens by indirect immunofluorescence. We identify a high frequency and wide range of clinically relevant autoantibodies in both acute and convalescent samples from COVID-19 patients. Their frequency and tissue specificity suggests that autoantibodies may contribute to the long-term consequences of COVID-19.

\section{Methods}

Participants Four cohorts of participants were recruited: the first group included 24 convalescent health care workers who had suffered from COVID-19 and not required hospitalisation to determine whether disease severity makes a difference to the generation of any autoantibodies. The second group included 25 individuals who were currently on the intensive therapy unit (ITU) diagnosed with COVID-19. The third group included 32 individuals who were currently on the ITU but for a reason other than COVID-19 to determine whether acute illness per se was associated with autoantibody production. The fourth group included 35 individuals who had been admitted to ITU with COVID-19 and had been discharged and their samples were taken at 3-6 month review. This group explored persistence of any potential antibodies.

Autoantibody assays Autoantibodies were detected on serum samples using indirect immunofluorescence. A spectrum of anti-neutrophil and organ specific autoantibodies were tested as a broad screen using standard ISO15189 accredited clinical tests. The immunoglobulin isotype detected was IgG with the exception of endomysial antibodies which are IgA. The full list of assays, manufacturers and disease association are described in theSupplementary data but in short we undertook indirect immunofluorescence on commercial pre-prepared slides to detect IgG antibodies for adrenal, autoimmune encephalitis, anti-neutrophil (ANA), anti-neutrophil cytoplasmic ANCA, cardiac, epidermal, islet cell, a range of cerebellar (Purkinje cell) antibodies, smooth muscle, mitochondrial, gastric parietal cell and skeletal muscle antibodies and IgA endomysial antibodies. All samples were read by two experienced clinical laboratory scientists for agreement. Data are largely descriptive. Inter group comparison was made by Chi Square using GraphPad Prism 9.

Ethical approval

For the acute cohort of ITU patient, surplus anonymised samples from routine clinical testing were used and for the convalescent ITU patients they were consented in clinic; ethical approval for these groups was granted by the North West-Preston Research Committee (ref 20/NW/0240 IRAS Project ID: 282164). The health care worker cohort was a random subgroup of COVID antibody positive patients from the COvid-19 COnvalescent immunity study (COCO) study approved by the London - Camden \& Kings Cross Research Ethics Committee reference 20/HRA/1817.

\section{Results}

There were 116 patient samples tested; 32 from group 1 (acute, non-COVID-19, ITU) 25 from group 2 (acute, COVID-19, ITU), 35 from group 3 (convalescent, COVID-19, post ITU) and 24 from group 4 (convalescent, COVID-19, non-hospitalised). The demographic descriptions inTable 1 find a female preponderance and slightly younger cohort in the non-hospitalised convalescent group. The average time from symptom onset was longer in the post ITU patients (151 days) than the non-hospitalised convalescent cohort (38 days). Non-white ethnicity is higher in all the COVID groups than the non-COVID ITU cohort, in keeping with known risk factors for severe COVID [14]. The details of number of samples tested are described in Table 
2 ; for some patients there was insufficient sample to run all tests.

The number of autoantibodies varied between groups. The highest numbers of autoantibodies to different antigenic targets was detected in the severe COVID disease groups. In group 1 (acute Non COVID-19, ITU) 13/32 (41\%) individuals had autoantibodies; 8 tested positive for 1 autoantibody, 4 for 2 autoantibodies and 1 for 3. For group 2 (acute COVID-19, ITU) 15/25 (60\%) individuals had autoantibodies; 12 tested positive for 1 autoantibody, 1 for 2 autoantibodies and 2 for 3. In group 3 (convalescent COVID-19, post ITU) 27/36 (75\%) individuals had autoantibodies, 14 tested positive for 1 autoantibody, 10 for 2 autoantibodies and 3 for 3. In group 4 (convalescent, COVID-19, non-hospitalised) 13/24 (54\%) individuals had autoantibodies and none tested positive for more than one (Figure 1 ).

Representative slides of epidermal, skeletal, cardiac and smooth muscle autoantibodies are shown in Figure 2 . In the acute Non COVID-19, ITU patients there were many different causes of their illness (Supplementary Table 1) and autoantibodies were found against nearly all $(12 / 13)$ of the different autoantigens examined, indicating a more random distribution. A higher proportion of acute COVID-19, ITU patients had autoantibodies $(60 \%$ versus $41 \%)$ but to a narrower range of autoantigens $(7 / 13)$ with a preponderance of epidermal (41\%) and skeletal antibodies (17\%). This preponderance was seen in convalescent, COVID-19, post ITU with epidermal (19\%) and skeletal antibodies (19\%) but additionally cardiac muscle antibodies $(28 \%)$ and smooth muscle antibodies (31\%). Representative slides of epidermal, smooth muscle, skeletal muscle and cardiac muscle autoantibodies are shown in

\section{Figure 2.}

In the convalescent, COVID-19, non-hospitalised cohort fewer individuals had autoantibodies than the convalescent ITU cohort (54\% versus $75 \%$ ) and against only four autoantigens: epidermal (25\%), smooth muscle (17\%), ANCA (8\%) and gastric parietal (4\%). These results suggest that COVID-19 infection is associated with autoantibody induction, and that these antibodies target a limited repertoire of self-antigens.

\section{Discussion}

SARS-CoV-2 is associated with a spectrum of illness during the acute viral infection, persisting during convalescence and as part of the long COVID syndrome. Constitutional, respiratory, cardiac, neurological, musculoskeletal and psychiatric symptoms are being increasingly described but the mechanisms behind these are uncertain [15]. It is not known whether these phenomena arise as direct effect of the virus or from off-target immune effects, including autoimmunity. Our study finds that there is a high prevalence of autoantibodies found in the acute and convalescent phase of COVID-19 suggesting that SARS-CoV-2 infection is associated with significant perturbations of immunological tolerance and raising the possibility that autoimmunity may play a role in the pathogenesis of acute and chronic symptoms.

Two fifths of the acute non COVID-19 ITU patients had autoantibodies suggesting that acute severe illness per se is associated with autoantibody production and the wide range of target autoantigens may reflect the diversity of this cohorts' underlying disease. Three fifths of the acute COVID-19, ITU patients had autoantibodies and these were of a narrower diversity with antibodies against epidermal intercellular cement and skeletal muscle predominant. These antibodies were persistent over time and also detected in convalescence post ITU COVID-19, over 5 months from symptoms onset. In addition, cardiac and smooth muscle antibodies were also identified. Cardiac and skeletal muscle autoantibodies were not found in convalescent individuals with non-hospitalised COVID-19, although smooth muscle antibodies were detected and a quarter had antibodies directed at epidermal intercellular cement. The narrow array of tissue specific autoantibodies associated with COVID-19 infection suggests loss of tolerance to these antigens may be disease specific, rather than a phenomenon associated with critical illness.

The link between infection and autoimmunity is well described with multiple genetic and environmental factors implicated [1]. Pathogenic mechanisms elucidated include molecular mimicry, epitope spreading, revelation of cryptic antigen and bystander activation, although which specific mechanism in which situation is usually uncertain. Similarly, just because an autoantibody is generated doesn't necessarily mean that 
the autoantibody is pathogenic. In some conditions such as myasthenia gravis there is a clear link between acetyl choline receptor antibodies and dysfunction of the motor end plate whereas in some conditions such as systemic lupus erythematosus the presence of high titre ANA is a non-specific biomarker of disease and the autoantibodies are not thought to be pathogenic. One of the limitations in understanding the role of autoantibodies in infectious disease has been the relative paucity of cases that are available to study within a reasonable time-frame. The sheer extent of the COVID-19 pandemic obviously overcomes this and work such as that presented here are first steps in interrogating these links.

The pattern of skin and muscle autoantibodies is intriguing and further studies are needed to elucidate the antigenic target and the clinical significance of these autoantibodies. One interesting possibility is the desmoglein family and DSG1 and 3 are found in the autoimmune blistering pemphigus disorders. Whilst oral ulceration and blistering has been described in COVID-19 $[16,17])$ it is by no means a commonly reported feature in large clinical studies such as ISARIC4C (Coronavirus Clinical Characterisation Consortium) [18].

This is an observational hypothesis generating clinical laboratory study that has raised the possibility of COVID infection generating a specific spectrum of autoantibodies. Determining the clinical relevance of these findings is still required but highlights specific history and examination that should be undertaken in COVID follow up clinics. Further studies examining the link between autoantibodies and COVID-19 are required to help understand how one virus can drive a myriad of presentations in different patients.

\section{Acknowledgements}

The authors would like to acknowledge the staff of the University of Birmingham Clinical Immunology Service for facilitating laboratory studies. The convalescent health care worker study COCO was carried out at the National Institute for Health Research (NIHR)/Wellcome Trust Birmingham Clinical Research Facility.

This study was funded as part of the UK Coronavirus Immunology Consortium funded by NIHR and UKRI. This paper presents independent research supported by the NIHR Birmingham Biomedical Research Centre at the University Hospitals Birmingham NHS Foundation Trust and the University of Birmingham.

\section{Competing interests}

MTD reports personal fees from Abingdon Health, outside the submitted work. All other authors declare no competing interests.

\section{References}

1. Kivity, S., et al., Infections and autoimmunity-friends or foes? Trends Immunol, 2009. 30 (8): p. 409-14.

2. Dalakas, M.C., Guillain-Barré syndrome: The first documented COVID-19-triggered autoimmune neurologic disease: More to come with myositis in the offing. Neurol Neuroimmunol Neuroinflamm, 2020.7 (5).

3. Sheikh, A.B., et al., Association of Guillain-Barre syndrome with COVID-19 infection: An updated systematic review. J Neuroimmunol, 2021. 355 : p. 577577.

4. Bhattacharjee, S. and M. Banerjee, Immune Thrombocytopenia Secondary to COVID-19: a Systematic Review. SN Compr Clin Med, 2020: p. 1-11.

5. Vlachoyiannopoulos, P.G., et al., Autoantibodies related to systemic autoimmune rheumatic diseases in severely ill patients with COVID-19. Ann Rheum Dis, 2020. 79 (12): p. 1661-1663.

6. Borghi, M.O., et al., Anti-Phospholipid Antibodies in COVID-19 Are Different From Those Detectable in the Anti-Phospholipid Syndrome.Front Immunol, 2020. 11 : p. 584241.

7. Galeano-Valle, F., et al., Antiphospholipid antibodies are not elevated in patients with severe COVID-19 pneumonia and venous thromboembolism. Thromb Res, 2020. 192 : p. 113-115.

8. Bastard, P., et al., Autoantibodies against type I IFNs in patients with life-threatening COVID-19. Science, 2020.370 (6515). 
9. Wang, E.Y., et al., Diverse Functional Autoantibodies in Patients with COVID-19. medRxiv, 2020.

10. Perez-Toledo, M., et al., Serology confirms SARS-CoV-2 infection in PCR-negative children presenting with Paediatric Inflammatory Multi-System Syndrome. medRxiv, 2020.

11. Consiglio, C.R., et al., The Immunology of Multisystem Inflammatory Syndrome in Children with COVID19. Cell, 2020.183 (4): p. 968-981.e7.

12. Gruber, C.N., et al., Mapping Systemic Inflammation and Antibody Responses in Multisystem Inflammatory Syndrome in Children (MIS-C). Cell, 2020. 183 (4): p. 982-995.e14.

13. Kreye, J., S.M. Reincke, and H. Prüss, Do cross-reactive antibodies cause neuropathology in COVID-19? Nat Rev Immunol, 2020.20 (11): p. 645-646.

14. Williamson, E.J., et al., Factors associated with COVID-19-related death using OpenSAFELY. Nature, 2020.584 (7821): p. 430-436.

15. Nalbandian, A., et al., Post-acute COVID-19 syndrome. Nat Med, 2021. 27 (4): p. 601-615.

16. Brandini, D.A., et al., Covid-19 and oral diseases: Crosstalk, synergy or association? Rev Med Virol, 2021.

17. Rekhtman, S., et al., Eruptions and related clinical course among 296 hospitalized adults with confirmed COVID-19. J Am Acad Dermatol, 2021. 84 (4): p. 946-952.

18. Swann, O.V., et al., Clinical characteristics of children and young people admitted to hospital with covid-19 in United Kingdom: prospective multicentre observational cohort study. Bmj, 2020.370 : p. m3249.

\section{Hosted file}

Figures.pdf available at https://authorea.com/users/376039/articles/519930-establishing-theprevalence-of-common-clinically-relevant-tissue-specific-autoantibodies-following-sarscov-2-infection

\section{Hosted file}

Tables.pdf available at https://authorea.com/users/376039/articles/519930-establishing-theprevalence-of-common-clinically-relevant-tissue-specific-autoantibodies-following-sarscov-2-infection

\section{Hosted file}

Supplementary data.pdf available at https://authorea.com/users/376039/articles/ 519930-establishing-the-prevalence-of-common-clinically-relevant-tissue-specificautoantibodies-following-sars-cov-2-infection 